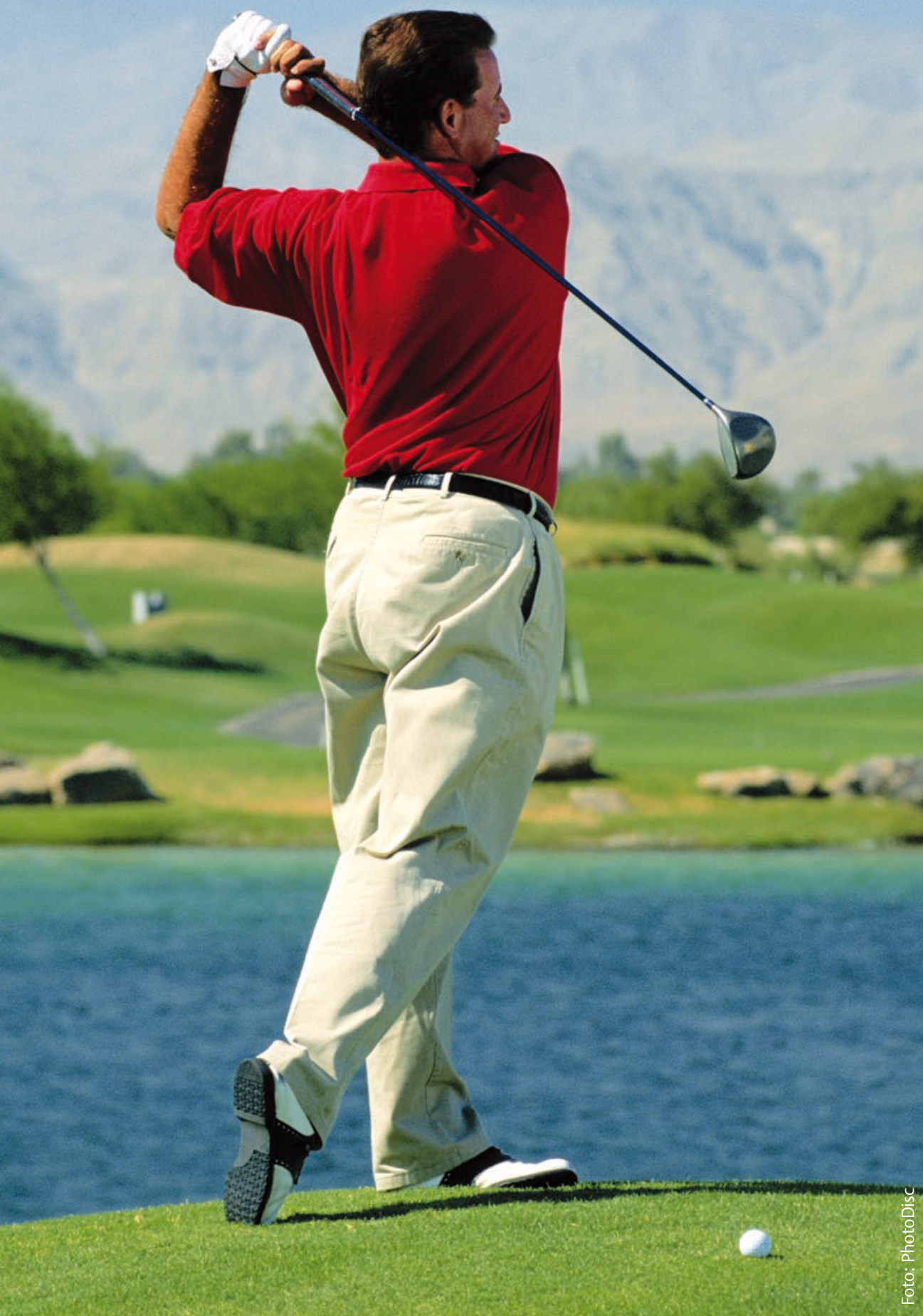

\section{Effektiv trainieren}

Neurologische Erkrankungen bringen häufig gravierende Einschränkungen der körperlichen Leistungsfähigkeit mit sich. Diese vermindern die Lebensqualität der betroffenen Patienten drastisch. Daher ist die Verbesserung der motorischen Leistungsfähigkeit durch Training ein zentrales Ziel rehabilitativer Maßnahmen bei neurologischen Patienten. Erkenntnisse aus der Sportwissenschaft bereichern hier das therapeutische Spektrum. Markus Gruber, Stefanie B. H. Gruber, Andreas Kramer

\section{Einleitung}

Die körperliche Leistungsfähigkeit des Menschen ist bedingt durch ein multifaktorielles und komplexes Zusammenspiel von physischen und psychischen Variablen. Dieses Zusammenspiel kann bislang erst ansatzweise und lediglich für einige Leistungskomponenten in seiner Komplexität verstanden werden. Aus diesem Grund existieren eine Vielzahl von Modellen, die versuchen, diese Komplexität mehr oder weniger zu reduzieren. Führt man die Modelle in ihrem Kern zusammen, kristallisiert sich ein Basismodell der körperlichen Leistungsfähigkeit heraus, das aus vier Komponenten besteht (s. Abb. 1).

\section{Intuitiv möchte man den Komponenten der körperlichen Leistungsfähigkeit - Konstitution, Kondition, Koordination und Psyche - einen hierarchischen Aufbau unterstellen, indem man die konstitutionellen Voraussetzungen als Basis sieht und darauf aufbauend konditionelle, dann koordinative und schließlich psychische Faktoren. In Wirklichkeit interagieren diese Systeme aber in komplexer Art und Weise, und wir wissen heute, dass eine Veränderung eines Subsystems immer auch Auswirkungen auf ein oder mehrere andere Subsysteme hat.}

Beispielsweise könnte ein Schlaganfall lediglich direkte Auswirkungen auf ein bestimmtes Hirnareal haben. In der Folge wird aber ein Teil der Skelettmuskulatur nicht mehr vollständig aktiviert, es kommt umgehend zu motorischen Störungen, schließlich zu Masseverlusten der betroffenen Muskeln und langfristig zum Abbau von Knochenmasse. Weiterhin sind häufig psychische Veränderungen zu beobachten, die ebenfalls wieder auf physische Komponenten rückkoppeln können.

Die Leistungsfähigkeit besitzt demnach neben einer genetischen auch eine verhaltensbedingte Ausprägung. Durch unser Verhalten, zu dem auch Krankheit und traumatische Ereignisse gezählt werden können, wirken wir spezifisch auf bestimmte Komponenten der Leistungsfähigkeit ein. Dies hat im Fall des oben beschriebenen Schlaganfalls weitreichende negative Auswirkungen. Im Fall eines zielgerichteten Trainings können im Gegensatz dazu positive Effekte erzielt werden. Unter Therapeuten gilt in der 


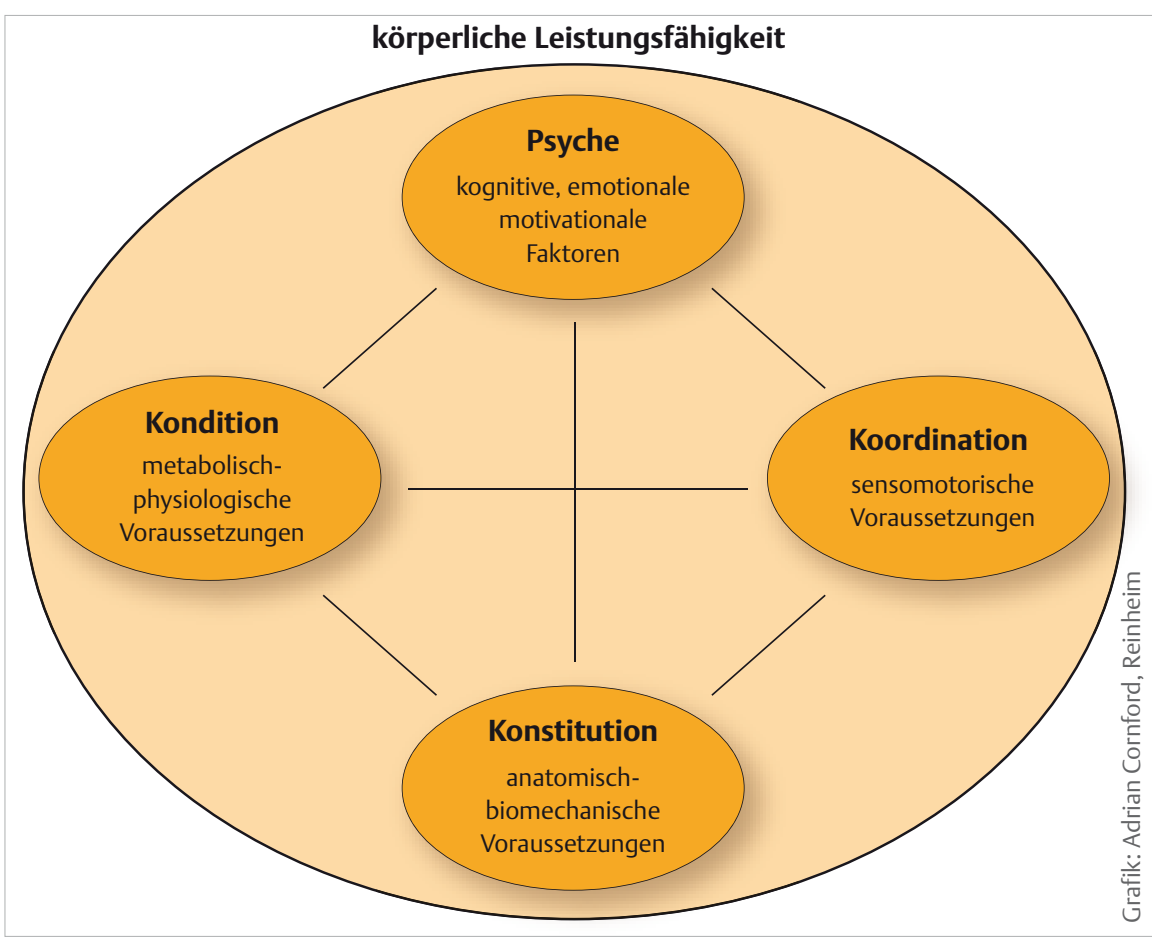

Abb. 1 Strukturmodell der körperlichen Leistungsfähigkeit. Die vier Komponenten Konstitution, Kondition, Koordination und Psyche stehen untereinander in Wechselwirkung und tragen in komplexer Weise gemeinsam zur körperlichen Leistungsfähigkeit bei.

neurologischen Rehabilitation für ein effektives Training häufig die Faustformel „repetitiv, intensiv, aufgabenspezifisch“. Der vorliegende Beitrag stellt dazu die wichtigsten Befunde aus der Trainings- und der Bewegungswissenschaft dar, die sich mit den konditionellen und koordinativen Leistungsvoraussetzungen beschäftigen.

Ziel ist es, einerseits Parallelen zu sportlichem Training zu beschreiben und andererseits Möglichkeiten aufzuzeigen, die bestehenden Trainingskonzepte in der Neurorehabilitation auf dem Hintergrund sportwissenschaftlicher Erkenntnisse weiterzuentwickeln.

\section{Biologische Anpassung als Grundlage von Training}

Um effektiv zu sein, muss Training eine Anpassungsreaktion auslösen, die zu einer möglichst großen Verbesserung der in s. Abb. 1 genannten Voraussetzungen der körperlichen Leistungsfähigkeit führt.

Regelkreis Die biologische Grundlage einer solchen Anpassung bildet ein geregeltes System, bestehend aus mindestens einem Messglied (Sensor oder Messfühler, erfasst Ist-Wert), einem Regler (Komparator, vergleicht Ist- und Soll-Wert) und einem Stellglied (wirkt der gemessenen Abweichung von Ist- zu Soll-Wert entgegen). Ein derartiger Regelkreis reagiert auf eine Störgröße, die von außen eingebracht wird, indem er eine Korrektur der Regelgröße bewirkt.

Als Beispiel soll an dieser Stelle die Anpassung der Knochenmasse angeführt werden. Die Deformation des Knochens ist hier die Regelgröße. Die Regelgröße muss in einem definierten Bereich liegen, damit Auf- und Abbauprozesse im Gleichgewicht (Homöostase) sind. Dies ist z. B. bei einem gesunden Menschen mit einer durchschnittlichen körperlichen Beanspruchung im Alltag der Fall. Wird der Mensch krank, erleidet er einen Unfall und bewegt sich weniger oder muss gar längere Zeit im Bett liegen, wirken geringere Kräfte auf den Knochen, und dieser wird in der Folge weniger stark deformiert. Mechanosensoren registrieren die Deformation und übermitteln diese Information an das zentrale Nervensystem. Von dort werden jetzt verstärkt knochenabbauende Zellen aktiviert und die Knochenmasse sinkt. Dieser Prozess wird so lange fortgeführt, bis die Regelgröße wieder im Sollbereich liegt [13]. Durch ein gezieltes Training, das z.B. begleitend während einer längeren Bettruhe über mehrere Wochen durchgeführt wird, kann der inaktivitätsbedingte Knochenabbau zum Teil verhindert werden [1].

\section{Training bedeutet demnach die Inanspruchnahme von Leistungsvoraussetzungen mit dem Ziel, eine Regelgröße systematisch zu beeinflussen und dadurch eine biologische Anpassung zu erreichen [39]. Training lässt sich folgendermaßen definieren: „Training ist die planmäßige und systematische Realisation von Maßnahmen (Trainingsinhalte und Trainingsmethoden) zur nachhaltigen Erreichung von Zielen (Trainingsziele) im und durch Sport“ [16].}

Trainingsreiz und biologischer Regellkreis Um das Training möglichst effektiv im Hinblick auf das zu erreichende Ziel planen zu können, müssten wir im Idealfall exakt die Wirkungen des Trainingsreizes im Hinblick auf die der gewünschten Anpassung zugrunde liegenden biologischen Mechanismen kennen. Leider sind aber weder die Mechanismen exakt bekannt noch die Verbindungen zwischen Trainingsreizen und biologischen Regelkreisen.

In der Trainingswissenschaft nimmt man aus diesem Grund eine zugängliche messbare Größe der körperlichen Leistungsfähigkeit, z.B. die maximale Kraft oder die maximale Sauerstoffaufnahme, und verwendet diese als Regelgröße. Der Messfühler ist die Leistungsdiagnostik, mit der über einen Test die maximale Kraft oder eben die maximale Sauerstoffaufnahme erfasst wird. Der Trainer oder Therapeut ist der Regler, der den Ist-Wert mit dem Soll-Wert abgleicht und auf Basis der gefundenen Abweichung das Training („Stellglied“) plant und durchführt.

\section{Training der konditionellen Voraussetzungen}

Je nach Sportart unterscheiden sich die Anforderungen an die konditionellen Voraussetzungen erheblich.

Leistungserbringung Der ausschlaggebende Unterschied ist die Leistung, die über einen bestimmten Zeitraum erbracht werden muss. Die mittlere Leistung und die Dauer, in der diese Leistung erbracht werden kann, sind dabei gegenläufig miteinander verbunden.

\section{Je länger der Zeitraum der Leistungserbringung, desto geringer die mögliche mittlere Leistung.}


Dieser Zusammenhang lässt sich recht einfach an folgendem Beispiel verdeutlichen: Gehen mit $5 \mathrm{~km} / \mathrm{h}$ ist für einen gesunden Menschen mittleren Alters stundenlang möglich, joggen mit $10 \mathrm{~km} / \mathrm{h}$ ungefähr eine Stunde, laufen mit $20 \mathrm{~km} / \mathrm{h}$ eine Minute und sprinten mit $30 \mathrm{~km} / \mathrm{h}$ $10 \mathrm{~s}$. Je schneller ich mich fortbewege, desto mehr Leistung muss ich erbringen. Biomechanisch bedeutet dies, dass ich mehr Energie in kürzerer Zeit zur Verfügung stellen muss.

Energiebereitstellung Hierfür ist in unserem Körper der Metabolismus (Stoffwechsel) zuständig. Unser Herz-Kreislauf-System versorgt die arbeitende Skelettmuskulatur und den Herzmuskel mit Sauerstoff, der in der Muskelzelle verwendet wird, um die Energieträger - Kohlenhydrate und Fette - zu verstoffwechseln. Freie Fettsäuren stehen uns in sehr großer Menge zur Verfügung und können uns stundenlang mit Energie versorgen. Die Stoffwechselprozesse sind aber langsam, und deshalb kann nur wenig Energie pro Zeit zur Verfügung gestellt werden; die Leistung ist gering. Die Speicherkapazität für Kohlenhydrate ist im Vergleich zu den Fetten deutlich geringer, die Energiebereitstellung erfolgt aber schneller. Steigt die Leistung, werden demnach immer mehr Kohlenhydrate verstoffwechselt. Stehen Kohlenhydrate nach z.B. einer Stunde nicht mehr in ausreichendem Maß zur Verfügung, kann die Leistung nicht mehr aufrechterhalten werden - die Laufgeschwindigkeit muss dann reduziert werden.

Sauerstoffverbrauch Für kurzzeitige Aktivitäten, bei denen sehr hohe Leistungen erforderlich sind, besitzen wir ein energielieferndes System, das zunächst ohne Sauerstoffverbrauch auskommt. Im Gegensatz zu den oben beschriebenen aeroben Energiegewinnungsprozessen nennt man es daher anaerobes System. Zunächst besteht die Möglichkeit, Glukose (gewonnen aus Kohlenhydraten) ohne Sauerstoff zu verstoffwechseln. Unter Sauerstoffmangel in der Muskelzelle wird Pyruvat, das durch die Glykolyse gewonnene Abbauprodukt der Glukose, außerhalb des Mitochondriums weiter zu Laktat abgebaut. Die Energiebereitstellung ist dabei gering, verläuft aber mit einer deutlich höheren Rate im Vergleich zu aeroben Prozessen. Die Leistung kann deutlich erhöht werden.

pH-Wert Allerdings entstehen bei den zugrunde liegenden biochemischen Prozessen $\mathrm{H}^{+}$-Ionen $(\rightarrow$ in wässrigem Milieu Hydroni-

Tab. 1 Laufstrecke und Weltrekordzeit geben den Umfang der sportlichen Leistungserbringung an. Die mittlere Laufgeschwindigkeit kann als Maß der mittleren Leistung angesehen werden. Je höher die Leistung ist, umso größer der anaerobe Anteil der Energiebereitstellung.

\begin{tabular}{|c|c|c|c|c|c|}
\hline Lauf & Zeit (WR) & $\begin{array}{l}\text { Mittlere Lauf- } \\
\text { geschwindigkeit }\end{array}$ & ATP/KrP & $\begin{array}{l}\text { Anaerob- } \\
\text { glykolytisch }\end{array}$ & Aerob \\
\hline Marathon & 2h 02’57“ & $20,5 \mathrm{~km} / \mathrm{h}$ & 5 & 5 & 90 \\
\hline $10000 \mathrm{~m}$ & $26^{\prime} 17^{\prime \prime}$ & $22,8 \mathrm{~km} / \mathrm{h}$ & 5 & 15 & 80 \\
\hline $5000 \mathrm{~m}$ & $12^{\prime} 37^{\prime \prime}$ & $23,8 \mathrm{~km} / \mathrm{h}$ & 10 & 20 & 70 \\
\hline $3000 \mathrm{~m}$ & 7’20“ & $24,5 \mathrm{~km} / \mathrm{h}$ & 20 & 40 & 40 \\
\hline $1500 \mathrm{~m}$ & 3'26" & $26,2 \mathrm{~km} / \mathrm{h}$ & 20 & 55 & 25 \\
\hline $800 \mathrm{~m}$ & 1'40“ & $28,8 \mathrm{~km} / \mathrm{h}$ & 30 & 65 & 5 \\
\hline $400 \mathrm{~m}$ & 43,18 & $33.3 \mathrm{~km} / \mathrm{h}$ & 80 & 15 & 5 \\
\hline $200 \mathrm{~m}$ & 19,19 & $37,5 \mathrm{~km} / \mathrm{h}$ & 95 & 2 & 3 \\
\hline $100 \mathrm{~m}$ & 9,58 & $37,6 \mathrm{~km} / \mathrm{h}$ & 95 & 3 & 2 \\
\hline
\end{tabular}

um-Ionen, $\mathrm{H}_{3} \mathrm{O}^{+}$), der $\mathrm{pH}-$ Wert in der Muskelzelle sinkt ab und das Zellmilieu übersäuert. Sinkt der pH-Wert immer weiter ab, werden für die Energiebereitstellung notwendige biochemische Reaktionen verhindert und die Leistung muss stark reduziert oder gar abgebrochen werden.

Phosphatverbindungen Für höchste Leistungen sind in der Muskelzelle hochenergetische Phosphatverbindungen gespeichert, wie das Adenosintriphosphat (ATP), das als primärer Energieträger direkt an der Kraftentwicklung beteiligt ist, und das Kreatinphosphat (KrP), das zur sehr schnellen Resynthese von ATP dient. Die Energiebereitstellungsrate ist bei diesen anaeroben alaktaziden Prozessen sehr hoch, was dazu führt, dass eine sehr hohe Leistung erbracht werden kann. Allerdings ist die Speicherkapazität im Muskel gering. Bei höchster Leistung sind die Phosphatspeicher deshalb nach ca. 10 s erschöpft.

\section{Zusammenhang mit der Laufleistung Zur} Verdeutlichung dieser metabolischen Grundlagen der körperlichen Leistung werden die Anteile der Energiebereitstellungssysteme in Tab. 1.1 in einen Zusammenhang mit der Leistung beim Laufen (hier approximiert durch die mittlere Laufgeschwindigkeit) und der Zeitdauer der Leistungserbringung gebracht [19].

Konditionelle Spezifität der Sportart Aus der Tabelle geht hervor, dass - in Abhängigkeit von der mittleren Leistung - die Anteile der beteiligten Energiebereitstellungssysteme unterschiedlich hoch sind. Die Leistung und die Zeitdauer, über die diese Leistung erbracht wird, bedingen im Wesentlichen die konditionelle Spezifität der Sportart. So zeichnen sich beispielsweise Sportlerinnen und Sportler, die über eine sehr lange Zeit ihre Leistung durch aerobe Energiebereitstellungsprozesse gewährleisten müssen, durch eine hohe maximale Sauerstoffaufnahmekapazität $\left(\mathrm{VO}_{2} \mathrm{max}\right)$ aus (Marathonläufer, Radfahrer, Triathleten, Skilangläufer). Während Sportlerinnen und Sportler, die über eine kurze Zeit sehr hohe Leistungen durch anaerobe Energiebereitstellungsprozesse gewährleisten müssen, in der Regel eine deutlich höhere Muskelmasse aufweisen (Sprinter, Turner, Judokas, Gewichtheber).

\section{In verschiedenen Sportarten sind je nach Anforderungsprofil der Sportart unterschiedliche Trainingsinhalte und Reizkonfigurationen erforderlich} (s. Abb. 2).

Trainingsintensität Dabei kommt der Trainingsintensität eine herausragende Bedeutung zu, denn sie bestimmt im Wesentlichen die abgerufene Leistung und damit den trainingswirksamen Reiz. Die Intensität muss für das Individuum relativ zur persönlichen Leistungsfähigkeit definiert werden. Ein biologischer Regelkreis pendelt sich nämlich immer wieder neu auf den aktuellen Leistungszustand einer Person ein und befindet sich dann in Homöostase. In diesem Zustand befinden sich aufbauende und abbauende Prozesse im Gleichgewicht, der Leistungszustand ändert sich nicht.

Ein trainingswirksamer Reiz muss auf die individuelle Leistungsfähigkeit (Regelgröße) bezogen sein, um für jedes Individuum eine ähnliche Anpassung zu erreichen. 


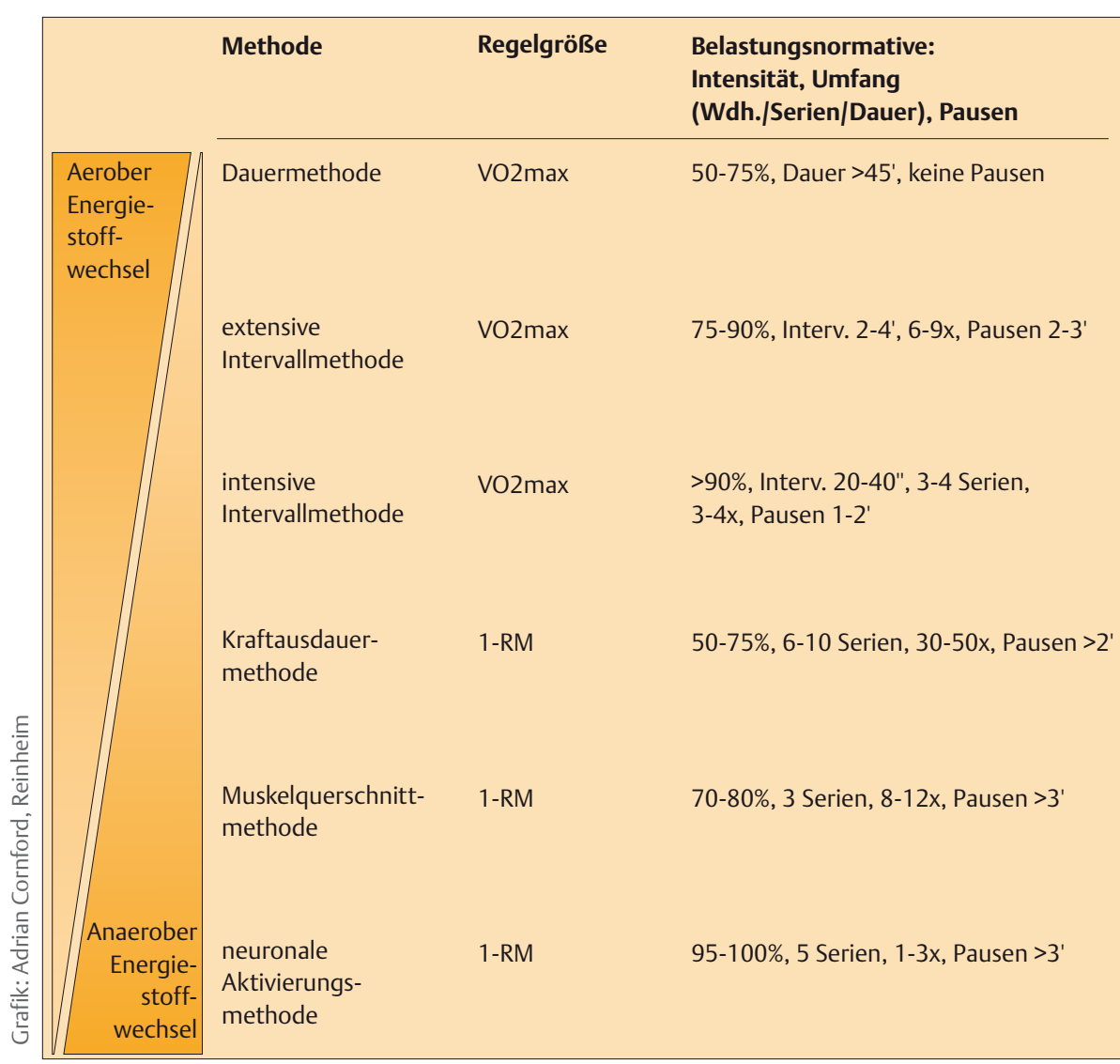

Abb. 2 Training der konditionellen Voraussetzungen anhand der Belastungsnormative Intensität, Umfang und Pausendauer. Über eine entsprechende Reizkonfiguration, bei der die Intensität individuell auf die Regelgröße abgestimmt sein muss, kann ein gezieltes Training der zugrunde liegenden Energiestoffwechselprozesse erzielt werden. Die sechs angeführten Methoden stellen die klassischen Trainingsmethoden mit weitgehend standardisierten Reizkonfigurationen dar.

Es ist offensichtlich, dass ein Olympiasieger über $800 \mathrm{~m}$ eine andere Geschwindigkeit laufen muss, wenn er die intensive Intervallmethode anwendet, als ein breitensportlich orientierter Läufer. Die Festlegung der Laufgeschwindigkeit für die beiden unterschiedlich leistungsfähigen Sportler gelingt über die Definition der Intensität relativ zum individuellen Leistungsmaximum.

Regelgrößen Als konkrete Regelgrößen bieten sich zur Verbesserung der aeroben Leistungsfähigkeit durch „Ausdauertraining“ die maximale Sauerstoffaufnahmekapazität (VO${ }_{2}$ max), die Laktatkonzentration im venösen Blut oder die Herzfrequenz und im therapeutischen Bereich auch die Selbsteinschätzung des Patienten über die Borg-Skala an [10]. Für die Trainingsgestaltung zur Verbesserung der anaeroben Leistungsfähigkeit wird für die Bestimmung der Intensität im „Krafttraining“ im Leistungssport das Einerwiederholungsmaximum („one repetition maximum“, 1-RM) direkt bestimmt, während im Gesundheitssport und in der Trainingstherapie das 1-RM häufig über die Anzahl der Wiederholungen mit submaximalen Gewichten berechnet wird [27].

Leistungsdiagnostik Die Bestimmung der oben angeführten Parameter erfolgt durch eine Leistungsdiagnostik. Beispielsweise können in einem Stufen- oder Rampentest auf einem Radergometer mittels Spirometrie, Laktatanalytik und Pulsmessung die Sauerstoffaufnahme, die Laktatkonzentration und die Herzfrequenz bei ansteigender Leistung gemessen werden. Daraus lassen sich anschließend die benötigten individuellen Vorgaben für die unterschiedlichen Trainingsbereiche ableiten (s. Abb. 2).

\section{Training der konditionellen Vorausset- zungen in der Neurorehabilitation} Patienten in der Neurorehabilitation besitzen im Allgemeinen deutlich geringere konditionelle Voraussetzungen im Vergleich zu gesunden Kontrollpersonen [33]. Nach dem Qualitätsgesetz sollte das niedrige Aus- gangsniveau eine schnelle und prozentual hohe Leistungssteigerung und damit eine gute Trainierbarkeit ermöglichen.

\section{In der Rehabilitation von neurologischen Patienten muss im Gegensatz zum Training mit Gesunden beachtet werden, dass die körperliche Leistungsfähigkeit beeinträchtigt ist, weil wichtige Teile des dafür zuständigen biologischen Systems geschädigt sind. Die Schädigung kann dabei akut traumatisch entstehen, wie z. B. beim Schlaganfall, oder sich chronisch entwickeln, wie z. B. bei Multipler Sklerose (MS). Darüber hinaus sind Komorbiditäten nicht selten. Eine Trainingsintervention muss deshalb in jedem Fall auf diese Ausgangsbedingungen abgestimmt werden. Grundsätzlich ist das biologische System aber auch nach Schädigung oder im Krankheitsverlauf plastisch und anpassungsfähig.}

Training bei MS Beispielhaft werden im Folgenden neuere Befunde zu trainingsbedingten Anpassungen bei MS vorgestellt. So konnten eine erhöhte maximale Sauerstoffaufnahmekapazität (13\%) und eine erhöhte Gangstrecke (60\%) nach aerobem Ausdauertraining nachgewiesen werden [32] sowie eine erhöhte willkürliche neuromuskuläre Aktivität (40\%) und eine größere Maximalkraft (20\%) nach einem intensiven Krafttraining [11]. In systematischen Überblicksartikeln und Metaanalysen konnten diese Effekte mit einem noch höheren Evidenzgrad belegt werden. Zusätzlich wurden Verbesserungen bei Alltagstätigkeiten, den „Activities of Daily Living (ADLs) berichtet, während insgesamt keine Evidenz für negative Effekte gefunden werden konnte [6].

Es ist darüber hinaus bekannt, dass körperliche Aktivität und sportliches Training nicht nur wichtige konditionelle Voraussetzungen der körperlichen Leistungsfähigkeit wie neuromuskuläre Kraft und Leistung oder die Ausdauer verbessern, sondern darüber hinaus das psychische und das soziale Wohlbefinden steigern können. Trainingstherapeutische Maßnahmen bei Multipler Sklerose unter Beachtung der krankheitsspezifischen Charakteristika (z.B. Überhitzung zu vermeiden) können daher empfohlen werden [24]. 
Das Beispiel „Multiple Sklerose“ eignet sich hierbei hervorragend, um den Stellenwert trainingswissenschaftlicher Erkenntnis zu verdeutlichen. Denn erst durch die Kenntnisse um individualisierte zielgerichtete und dadurch effektive Trainingsinterventionen konnten in grundlegenden wissenschaftlichen Studien die notwendigen positiven Befunde generiert werden, um diese Intervention zu etablieren und vorhandene Bedenken gegen ein körperliches Training bei diesem Krankheitsbild auszuräumen.

\section{Es darf an dieser Stelle nicht vergessen werden, dass sich Plastizität und Anpassungsfähigkeit nicht nur darauf bezieht, Leistungsvoraussetzungen durch Training zu verbessern, sondern auch diese durch inaktives Verhalten nach dem Prinzip „use it or lose it“ zu mindern.}

Bettruhestudien Inaktivität kann damit zu einem Hauptproblem der Patienten werden. Wir wissen aus sogenannten Bettruhestudien, dass während körperlicher Inaktivität selbst bei Gesunden alle Leistungsparameter drastisch zurückgehen. Die maximale Leistung der Beinmuskulatur etwa geht nach achtwöchiger Bettruhe bei Gesunden um ca. $25 \%$ zurück [2] und die maximale Sauerstoffaufnahmekapazität $\mathrm{VO}_{2}$ max bereits nach zwei Wochen um ca. 10\% [5]. Lediglich sehr intensive Trainingsinterventionen sind in der Lage, bei einem Teil der Parameter (z. B. $\mathrm{VO}_{2} \mathrm{max}$ ) die Abnahme zu verhindern. So sollten z. B. Astronauten und Kosmonauten, die sich in Schwerelosigkeit auf der internationalen Raumstation (ISS) aufhalten, ca. 4 Stunden pro Tag trainieren, um die inaktivitätsbedingten Verluste der aeroben und anaeroben Kapazität in annehmbaren Grenzen zu halten [45].

Intensives Training Es ist davon auszugehen, dass bei einem Patienten, der z. B. einen Schlaganfall erlitten hat, neben der direkten Schädigung, die zu motorischen Einschränkungen und einer verminderten körperlichen Leistungsfähigkeit führt, diese weiter durch die liegende oder sitzende Haltung ohne körperliche Aktivität negativ beeinflusst wird. In so einem Setting finden aus sportwissenschaftlicher Sicht therapeutische Maßnahmen oft schlicht in einem zu geringen Umfang statt, um effektiv einer inaktivitätsbedingten Verschlechterung der körper- lichen Leistungsfähigkeit entgegenwirken zu können [25]. Um dem zu begegnen, wird in der Neurorehabilitation in neueren Publikationen oft ein intensives Training („intensive practice“) eingefordert [47, 20]. Gemeint ist aber hier insbesondere der Trainingsumfang, also die Zeit, die z. B. pro Tag trainiert wird, und weniger die Trainingsintensität, also die Leistung in Relation zur maximalen Leistung, bei der trainiert werden kann.

\section{Ein umfangbetontes Training sollte möglichst frühzeitig im Verlauf der Rehabilitation - z. B. nach Schlaganfall \\ - erfolgen, um die ablaufenden \\ Regenerationsprozesse zu fördern und zu verhindern, dass diese durch Inaktivität behindert oder sogar verhindert werden.}

\section{- Training der koordinativen Voraussetzungen}

Sportlerinnen und Sportler aus Sportarten, die ein sehr vergleichbares konditionelles Anforderungsprofil aufweisen, besitzen durchaus auch vergleichbare basale konditionelle Voraussetzungen (z.B. eine vergleichbar hohe $\mathrm{VO}_{2} \max$ ).

Sportartspezifische Leistung Trotz einer vergleichbar hohen aeroben Kapazität wird ein Marathonläufer eine deutlich geringere Leistungsfähigkeit in anderen Sportarten im Vergleich mit den jeweiligen Sportartspezialisten, wie z.B. mit einem Radfahrer, Ruderer oder Skilangläufer, aufweisen. Grund sind koordinative Voraussetzungen, die sogenannte sportartspezifische Technik, die zusammen mit den konditionellen, konstitutionellen Voraussetzungen und den psychischen Faktoren im Wesentlichen die sportartspezifische Leistung determinieren (s. Abb. 1). Je größer der Einfluss der sportlichen Technik wird, umso geringer wird der Übertrag in eine andere Sportart. Turner, Judokas und Gewichtheber weisen alle eine ausgeprägte anaerobe Leistungsfähigkeit auf, können diese aber aufgrund der sportartspezifischen technischen Anforderungen kaum in die anderen genannten Sportarten übersetzen. In diesem Zusammenhang spricht man umgangssprachlich gerne von Sportartspezialisten.

Bewegungsfertigkeit Die Bewegungsfertigkeit, die einer sportlichen Technik zugrunde liegt, zu definieren, ist nicht trivial. Schmidt und Lee [38] definieren eine motorische Fertigkeit auf der Verhaltensebene. Es ist für sie die „Fähigkeit, ein Bewegungsziel mit maximaler Präzision und minimalem Energie- und Zeitaufwand zu erreichen" (übersetzt durch die Verfasser). In der Motorikforschung zielen die Definitionen stärker auf die motorische Ausführungsebene ab. Nach Kitago und Krakauer [20] ist es beispielsweise die „Fähigkeit, eine genaue Bewegungsausführung verlässlich zu reproduzieren“.

Bewegungsgeschwindigkeit Als wichtiges Charakteristikum ist festzuhalten, dass die Präzision der Bewegung oder die Geschwindigkeit der Bewegung allein jeweils kein Kriterium für die Qualität der Bewegungsfertigkeit darstellen. Geschwindigkeit und Präzision stehen in einem inversen Verhältnis zueinander. So erhöht sich die Fehlerrate, wenn Studienteilnehmer aufgefordert werden, die Bewegungsgeschwindigkeit zu erhöhen, und die Bewegungsgeschwindigkeit wird reduziert, wenn sie aufgefordert werden, die Bewegung präziser auszuführen.

\section{Um bei einer Bewegungsfertigkeit eine hohe Präzision in Kombination mit einer hohen Bewegungsgeschwindigkeit in einer sportlichen Technik, wie z. B. einem Wurf im Basketball, einem Salto im Turnen oder einem Rückhandschlag im Tennis, zu erlangen, muss die Bewegungsausführung zunächst erlernt und dann in jahrelangem Techniktraining perfektioniert werden.}

Motorisches Lernen: Definition Eine Definition von motorischem Lernen ist ähnlich schwierig zu formulieren wie die der Bewegungsfertigkeit selbst, da das Lernen an sich nicht beobachtbar ist. Schmidt und Lee [38] kommen aufbauend auf den Charakteristiken von Lernprozessen $\mathrm{zu}$ folgender Definition (übersetzt durch die Verfasser): „Motorisches Lernen ist die Summe der Anpassungsprozesse, die durch Training oder Erfahrung ausgelöst werden und zu relativ dauerhaften Veränderungen von Bewegungsfertigkeiten führen.“

Motorische Lernphasen Beobachtet man den Lernprozess einer Bewegung (z.B. eines Golfschlags) von der ersten Trainingseinheit bis zur Beherrschung in Perfektion, dann erscheint dieser in abgrenzbaren Pha- 
sen zu verlaufen [12]. Meinel und Schnabel [30] haben aus einer methodisch-didaktischen Sichtweise den langfristigen Lernprozess beim Technikerwerb in Anlehnung an die bereits vorhandenen Einteilungen in drei motorische Lernphasen unterteilt:

- In der ersten Phase, die als Grobkoordination bezeichnet wird, kann die Bewegungsaufgabe nur bei sehr günstigen Bedingungen gelöst werden, wobei die Bewegungsausführung dem Technikleitbild lediglich grob entspricht.

- In der zweiten Phase, der Feinkoordination, kann die Bewegungsaufgabe bei normalen Bedingungen ohne Probleme gelöst werden. Die Bewegung entspricht dabei dem Technikleitbild und genügt den Bedingungen einer gut koordinierten Bewegung.

- In der dritten Phase, der Feinstkoordination oder Feinkoordination unter variablen Bedingungen, kann die Bewegungsaufgabe auch unter erschwerten Bedingungen mit großer Sicherheit und Konstanz erfüllt werden. Bei sportlichen Bewegungen, die hohe Anforderungen an koordinative oder auch konditionelle Voraussetzungen stellen, sind dafür teilweise 10 Jahre oder mehr nötig (z.B. Golf), bei manchen Bewegungsfolgen ist eine so hohe Bewegungswiederholung oftmals überhaupt nicht zu erreichen ( $z$. B. Skisprung). Bei Alltagsaktivitäten (Essen mit Besteck) oder zyklischen Bewegungen (Radfahren, Laufen etc.) schaffen wir dagegen im Laufe des Erwachsenwerdens ohne Probleme eine entsprechend hohe Anzahl an Bewegungswiederholungen, die es uns erlaubt, in den Bereich der Feinstkoordination zu kommen.

Power Law of Practice Zugrunde liegt das „Power Law of Practice“. Dieses Gesetz besagt, dass der Übungsumfang, also die Anzahl an Bewegungswiederholungen, den Lernerfolg determiniert, wenn die Übungsbedingungen vergleichbar sind. Die Lernkurve verläuft dabei nicht linear ansteigend, sondern zeigt ein beschränktes Wachstum. Zu Beginn ist deshalb der messbare Leistungszuwachs deutlich größer als in einem fortgeschrittenen Lernstadium.

In einer klassischen Studie aus dem Jahr 1959 konnte Crossman [38] nachweisen, dass die Zeit, die Arbeiter benötigten, um eine Zigarre herzustellen, von der Anzahl an bereits hergestellten Zigarren und damit vom
Übungsumfang der Arbeiter abhängig war. So lag die mittlere Produktionszeit für eine Zigarre nach 10.000 hergestellten Zigarren bei ca. 25 s, nach 100.000 hergestellten Zigarren bei ca. $13 \mathrm{~s}$, nach 1.000 .000 Zigarren bei ca. 9s und nach 10.000.000 Zigarren bei ca. $7 \mathrm{~s}$. Später durchgeführte Untersuchungen an Violinisten unterschiedlicher Könnensstufen, von Violinschülern und Violinlehrern bis hin zu Profimusikern, bestätigen die herausragende Stellung des hochrepetitiven Übens [9].

Aus dem „Power Law of Practice“ und
der aktuellen Studienlage lässt sich
schlussfolgern, dass der Übungsumfang
die absolut wichtigste Variable ist, die
das Lernen beeinflusst. Das effektivste
Vorgehen zur Verbesserung einer
motorischen Fertigkeit scheint
demnach eindeutig die Steigerung
der Wiederholungszahl in einer
Trainingseinheit zu sein [20].

Steigender Übungsumfang Die dem Lernen hinterlegte exponentielle Gesetzmäßigkeit hat dabei für die Bewegungsfertigkeit zwei direkte praktische Auswirkungen. Zu Beginn des Erlernens einer Bewegung sind schnelle Fortschritte erkennbar; um aber die koordinative Leistung immer weiter zu steigern, muss mit steigendem Leistungsniveau ein immer größerer Übungsumfang erfolgen. Spitzenleistungen in technisch anspruchsvollen Sportarten erfordern daher eine mehrjährige Technikerwerbsphase und anschließend ein tägliches Training, denn bereits nach wenigen Tagen ohne Üben der Bewegungsfertigkeit können aufgrund der vorhandenen Plastizität bereits Einbußen in der Präzision und Geschwindigkeit der Bewegungsausführung entstehen.

Beschleunigung des Lernprozesses Um Lernmethoden und Lernbedingungen zu entwickeln, die es ermöglichen, den Lernprozess unabhängig von der Übungshäufigkeit zu beschleunigen, sind in den vergangenen Jahrzehnten eine Vielzahl von wissenschaftlichen Studien durchgeführt worden. Die wichtigsten Erkenntnisse werden im folgenden Abschnitt vorgestellt und diskutiert.

Kontext-Interferenz-Effekt Eine Lernbedingung, die sehr umfangreich untersucht wurde, ist der Einfluss der Übungsabfolge auf die Lernleistung, bekannt unter dem Na- men „Kontext-Interferenz-Effekt“ (contextual interference). Sollen in einer Trainingseinheit mehrere Bewegungsfertigkeiten (z.B. A, B , C) trainiert werden, stellt sich die Frage, wie die beste Übungsabfolge dazu aussieht. Konkret: Soll die Übungsabfolge blockweise erfolgen (z. B. A, A, A; B, B, B; C, C, C) oder verteilt (z.B. A, C, B, C, A, B).

In der blockweisen Methode wird ein und dieselbe Bewegungsaufgabe mehrmals hintereinander ausgeführt, während in der verteilten Methode immer unterschiedliche Bewegungsaufgaben aufeinander folgen.

\section{Die Studienlage hierzu ist eindeutig: Verteiltes Trainieren ist dem geblockten Trainieren überlegen.}

Dieses Ergebnis ist nicht trivial und wird in der Praxis häufig nicht beachtet. Der Grund hierfür liegt sehr wahrscheinlich darin, dass häufig fälschlicherweise die Verbesserung in der Bewegungsfertigkeit während der Aneignungsphase (in einer Übungseinheit) als Lernleistung herangezogen wird und nicht die Verbesserung in der Bewegungsfertigkeit zwischen zwei Trainingseinheiten (Behaltensleistung). Genau diese Behaltensleistung (Retention) lässt aber Rückschlüsse auf das zugrunde liegende motorische Lernen zu (s. Definition).

In Abb. 3 sind die Ergebnisse von Studien zu Kontext-Interferenz-Effekten beim motorischen Lernen schematisch dargestellt [41].

Diese Darstellung eignet sich, um folgende Punkte herauszuarbeiten:

- Die Leistungszunahme in der Bewegungsfertigkeit während einer Trainingseinheit (Aneignungsphase) spiegelt nicht zwangsweise die Leistungszunahme zur nächsten Trainingseinheit wider (Behaltensleistung, Retention).

- Der Kontext-Interferenz-Effekt verstärkt das motorische Lernen. Verteiltes Trainieren ist geblocktem Trainieren überlegen.

- Insbesondere gilt dies, wenn die Leistungserbringung nicht geblockt abgerufen werden soll. Beispielsweise wird im Golf während eines Wettkampfs in der Regel niemals zweimal hintereinander der gleiche Schläger verwendet, geschweige denn der gleiche Schlag gespielt oder zwei Bälle von einer ähnlichen Stellung geputtet. Dies gilt im Übrigen auch für Alltagstätigkeiten, z. B. beim Essen, im Haushalt oder beim Anziehen. 


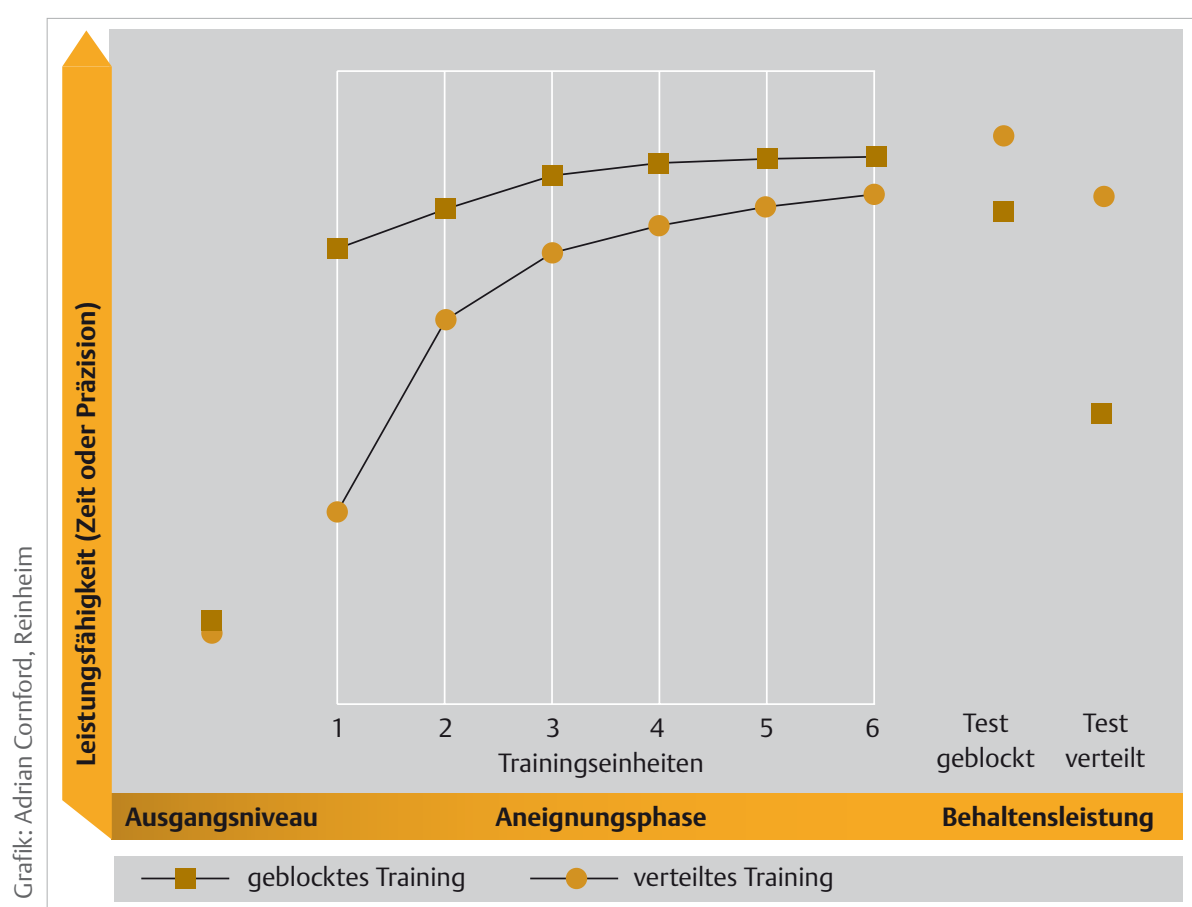

Abb. 3 Schematische Darstellung der Entwicklung der motorischen Leistungsfähigkeit in einer Fertigkeitsaufgabe bei geblocktem Trainieren gegenüber verteiltem Trainieren. Es kann von einer höheren Leistungsfähigkeit ausgegangen werden, wenn z. B. die Aufgabe in kürzerer Zeit erledigt wird oder präziser. Während der Trainingseinheiten kommt es zu einer Verbesserung der Leistungsfähigkeit in beiden Gruppen. Diese fällt bei geblocktem Trainieren zu Beginn der Aneignungsphase deutlich größer aus als bei verteiltem Trainieren. Nach einigen Tagen jedoch kehrt sich das Ergebnis um. Die Behaltensleistung ist generell und insbesondere für den Test in einer verteilten Aufgabenstellung deutlich größer für die Gruppe, die verteilt trainiert hat, im Vergleich zu der Gruppe, die geblockt trainiert hat. Da vor dem Training ein vergleichbares Ausgangsniveau beider Gruppen gegeben war, kann man davon ausgehen, dass verteiltes Trainieren im Vergleich zu geblockten Trainieren zu einem größeren Lernerfolg führt.

Anforderungen des Lernsettings Der Mensch ist darauf spezialisiert, zu lernen und insbesondere motorisch zu lernen. Dabei stellt er sich perfekt auf die Anforderungen des Lernsettings ein. Werden z. B. beim Golftraining mehrere Bälle von derselben Stelle in der geblockten Trainingsform auf das Loch geschlagen oder geputtet, dann muss der Spieler lediglich den ersten Schlag ohne ein direktes Feedback planen. Für den zweiten Schlag steht ihm das Feedback, das er während und nach dem ersten Schlag erhalten hat, zur Verfügung, für den dritten Schlag das Feedback aus dem ersten und zweiten usw. [4]. Der Spieler, der geblockt trainiert, wird sicher im Vergleich zu einem Spieler, der verteilt trainiert, viel besser in der Lage sein, Feedback aus einem Schlag für einen nachfolgenden identischen Schlag zu nutzen, allerdings wird er diese Fertigkeit leider im Spiel niemals anwenden können. Dagegen muss der Spieler, der verteilt übt, jeden Schlag in der Trainingseinheit neu planen; sein Fortschritt ist in- nerhalb der Einheit scheinbar geringer, seine Behaltensleistung bis zur nächsten Trainingseinheit ist aber größer, er hat „mehr gelernt" [34].

Feedback Im vorigen Absatz ist bereits Feedback als ein weiterer entscheidender Lernmechanismus angesprochen worden. Intrinsisches Feedback erlaubt es uns, die Bewegungsleistung mithilfe unserer sensorischen Systeme zu bewerten. Bei einem BasketballFreiwurf sieht der Spieler beispielsweise, ob der Ball in den Korb gegangen ist, und kann den Ballflug beobachten, der zum Erfolg oder Misserfolg des Wurfs geführt hat.

Extrinsisches Feedback oder „Von außen verstärkendes“ (augmented) Feedback kann zusätzliche Informationen zum Bewegungsergebnis (Knowledge of Result, KR) oder zum Bewegungsablauf (Knowledge of Performance, KP) bereitstellen. In der Regel wird dieses Feedback vom Trainer während der Trainingseinheit oder auch in Form einer Videoanalyse nach einer Trainingsein- heit oder einem Wettkampf gegeben. Während ganz ohne Feedback Lernen grundsätzlich unmöglich ist, gibt es unterschiedliche Studienergebnisse zur „optimalen Menge“ an Feedback, insbesondere des von außen gegebenen zusätzlichen Feedbacks.

Die Mehrzahl der Studien zeigen, dass zusätzliches von außen gegebenes Feedback den Lernprozess generell beschleunigt, dass es aber durch massives zusätzliches Feedback über einen längeren Zeitraum zu einer Abhängigkeit von diesem Feedback kommen kann. Es wird daher empfohlen, das zusätzliche Feedback mit der Zeit abzubauen [46].

Ebenso spielt der Zeitpunkt, an dem der Lernende das Feedback von außen bekommt, eine entscheidende Rolle. Es konnte gezeigt werden, dass ein sofortiges Feedback unmittelbar nach der Bewegung nicht zu vergleichbar guten Lerneffekten führte wie ein leicht verzögertes Feedback, das einige Sekunden nach Beendigung der Bewegung gegeben wurde [43].

Aufmerksamkeitsfokus Neben dem Feedback spielt der Aufmerksamkeitsfokus eine wichtige Rolle. Worauf achten wir z. B., wenn wir im Training einen Freiwurf im Basketball oder einen Golfschlag ausführen? Worauf sollten wir unsere Aufmerksamkeit richten, um möglichst effektiv zu lernen? Zunächst können wir grundsätzlich zwei Aufmerksamkeitsfokusse unterscheiden: Aufmerksamkeit kann internal und external gerichtet sein. Bei einem internalen Fokus wird die Aufmerksamkeit auf sensorische oder motorische Signale des eigenen Körpers gerichtet, bei einem externalen Fokus auf ein Ereignis außerhalb des Körpers, das mit dem Bewegungsergebnis in Verbindung steht. Beispielsweise kann die Aufmerksamkeit bei einem Golfschlag auf die Bewegung des Armes gerichtet sein oder auf die Bewegung des Schlägerkopfs, beide Male mit dem Ziel, einen Ball möglichst nahe an ein Ziel zu schlagen [48].

In einer Vielzahl von Experimenten dieser Art bei unterschiedlichsten sportlichen Bewegungen und Gleichgewichtsaufgaben konnte 
eindeutig gezeigt werden, dass ein externaler Aufmerksamkeitsfokus einem internalen Fokus sowohl im Hinblick auf die motorische Leistung als auch die Lerngeschwindigkeit überlegen ist [49].

Instruktion Obwohl die Ergebnisse auf der Verhaltensebene einen hohen Evidenzgrad aufweisen, sind die zugrunde liegenden Mechanismen noch nicht geklärt. Es wird vermutet, dass es durch einen internalen Fokus zu Überlagerungen mit automatisierten Bewegungsprogrammen kommt, der diese stört, während dies bei einem externalen Fokus nicht der Fall ist [26]. Für den Trainer bzw. die Therapeutin besitzen diese Befunde eine wichtige Konsequenz, da er oder sie in der Lage ist, die Aufmerksamkeit des Lernenden über eine Instruktion zu lenken. Diese Instruktion oder Bewegungsanweisung sollte, um effektives Lernen zu ermöglichen, die Aufmerksamkeit external attribuieren.

Geführtes Training Wie geht man am besten vor, wenn der Lernende die Bewegung noch gar nicht beherrscht oder sehr unpräzise Bewegungsvorstellungen besitzt? Ist dann ein geführtes Training von Vorteil? Aus dem Sport liegen hierzu mehrere Studien vor, die übereinstimmend einen positiven Effekt während der Aneignungsphase zeigen (bessere Leistung und größere Leistungszuwächse), aber einen deutlich schlechteren Effekt für die Retention [26]. Eine von außen geführte Bewegung wirkt sich nach diesen Erkenntnissen negativ auf die Behaltensleistung und damit den motorischen Lernvorgang aus. Erklärt wird dies durch den Umstand, dass sich der Lernende auf die Führung verlässt und daher die sehr gute Aneignung, aber nur mäßige Retention zu beobachten ist [15]. Dieses Verhalten ist mit den Ergebnissen der FeedbackForschung vergleichbar (s.o.), die ebenfalls zeigen, dass ein während der Bewegung gegebenes Feedback oder ein unmittelbar gegebenes Feedback weniger effektiv ist als ein leicht verzögertes Feedback. Dennoch besitzt die z.B. robotergeführte Bewegung möglicherweise Vorteile in einer sehr frühen Phase des motorischen Fertigkeitserwerbs, in der das Bewegungsprogramm noch nicht existiert und sozusagen erst einmal vorprogrammiert werden muss [23].
Transfereffekt Kann das Erlernen einer spezifischen motorischen Fertigkeit zu einem Transfereffekt und damit zu einer verbesserten Leistung in einer anderen motorischen Fertigkeit führen?

\section{Das Erlernen einer spezifischen motorischen Fertigkeit kann zu einem Transfereffekt führen, aber nur, wenn die Fertigkeiten beinahe identisch sind - und selbst dann sind die Transfereffekte in der Regel klein [37].}

Das grundlegende Konzept wurde zum ersten Mal bereits vor über 100 Jahren von Thorndike und Woodworth [44] vorgestellt. Die beiden Autoren postulierten, dass die Höhe des Transfers zwischen zwei Bewegungsfertigkeiten von der Anzahl „identischer Elemente“ abhängig wäre. In der Zwischenzeit wurden etliche Untersuchungen durchgeführt, die diese Grundannahme im Wesentlichen bestätigt haben (s. hierzu auch den Beitrag von Huber in diesem Heft [18]).

Erst kürzlich ist es uns gelungen, dies auch für Gleichgewichtsaufgaben zu zeigen: Eine Gruppe trainierte eine Gleichgewichtsaufgabe, die andere Gruppe eine zweite Gleichgewichtsaufgabe. Nach dem Training war jede Gruppe in der jeweils von ihr trainierten Aufgabe deutlich besser, aber in den Gleichgewichtsaufgaben, die sie nicht trainiert hatten, waren keine signifikanten Verbesserungen zu sehen [14].

Diese Befunde entsprechen weitgehend auch der Tatsache, dass nach Gleichgewichtstraining hochspezifische und fertigkeitsbezogene neuronale Veränderungen gefunden werden konnten [40].

\section{Gleichgewichtstraining kann durchaus als Fertigkeitstraining und sensomotorischer Lernprozess aufgefasst werden.}

Training der koordinativen Voraussetzungen in der motorischen Neurorehabilitation

Die motorische Neurorehabilitation baut im Wesentlichen auf der Annahme auf, dass eine Verbesserung von Bewegungsfertigkeiten auch bei neurologischen Patienten durch Trainingsmaßnahmen möglich ist [21]. Diese Annahme ist nicht selbstverständlich, da durch eine neurologische Schädigung oft auch Strukturen in Mitleidenschaft gezo- gen sind, die direkt mit motorischen Lernvorgängen in Verbindung gebracht werden, wie z.B. Kleinhirn, Basalganglien, prämotorischer und motorischer Kortex oder auch parietaler Assoziationskortex.

Tatsächlich gibt es Hinweise darauf, dass es bei bestimmten Schädigungen, z. B. im Bereich des Kleinhirns, zu Defiziten bei motorischem Lernen gegenüber gesunden Vergleichspersonen kommen kann. Allerdings ist die Studienlage nicht eindeutig. Eine starke Beeinträchtigung der Lernfähigkeit scheint bei Patienten in der Regel aber nicht gegeben zu sein [20]. Eine Schwierigkeit, dies zu untersuchen, besteht darin, das Wieder- oder Neulernen von Bewegungsfertigkeiten von parallel dazu ablaufenden Regenerationsprozessen abzugrenzen. Darüber hinaus kommt es im Laufe der Rehabilitation oft zu Kompensationsreaktionen, die ebenfalls nicht eine Verbesserung der ursprünglichen Bewegungsfertigkeit widerspiegeln.

\section{Viele der klinischen Tests sind nicht unmittelbar brauchbar, um die Verbesserung der Bewegungsfertigkeit per se zu messen, weil sie oft nur outcomeorientiert sind und die Bewegungsqualität explizit nicht einbeziehen. Eine moderne motorischen Neurorehabilitation sollte hier nicht nur danach fragen, ob eine Intervention wirkt, sondern welche Mechanismen zur Verbesserung der Bewegungsleistung beitragen.}

Noch vorhandene Leistungsvoraussetzungen Erste Experimente dazu wurden bereits am Tiermodell durchgeführt. In einem „Ratten-Schlaganfall-Modell“ konnten dabei Interaktionseffekte von spontanen Wiederherstellungsprozessen und sensomotorischem Training gezeigt werden [31]. Diese Untersuchungen unterstützen erste Indizien, die nahelegen, dass eine möglichst frühe „Inanspruchnahme von noch vorhandenen Leistungsvoraussetzungen“, z. B. nach einem Schlaganfall, von entscheidender Bedeutung für den Rehabilitationsprozess im Gesamten sein könnte. Die „Constraint-Induced Movement Therapy“ (CIMT) und die Forced-Use-Therapie greifen dieses Paradigma auf und zwingen den Patienten beispielsweise zur Nutzung des „betroffenen“ Arms, indem der „nicht betroffene“ Arm in 
seiner Bewegungsamplitude artifiziell eingeschränkt wird und deshalb seine Funktion nicht mehr wahrnehmen kann $[28,7]$.

Robotergestützte Therapie Im Grundsatz beziehen sich diese Ansätze auf das ,power law of practice“, indem sie den Patienten dazu zwingen, Zielbewegungen bzw. Bewegungsabläufe wiederholt durchzuführen. Diesen Ansatz greift auch die robotergestützte Therapie auf. Durch geführte Bewegung kann auch ein konditionell und koordinativ stark betroffener Patient eine hohe Anzahl an Bewegungsabläufen absolvieren. Problematisch sind in diesem Zusammenhang die schon angeführten negativen Befunde zum geführten Training im Hinblick auf die Lernleistung. Für die Neurorehabilitation liegen allerdings sowohl für die untere Extremität als auch für die obere Extremität Befunde vor, die der roboterassistierten Therapie eine therapeutische Wirksamkeit bescheinigen [29]. Diese therapeutische Wirksamkeit ist vermutlich dadurch bedingt, dass durch das niedrige Ausgangsniveau der Patienten und den ausschließlich mit dieser Therapie möglichen hohen Trainingsumfang (hohe Anzahl an Bewegungswiederholungen) eine Verbesserung der Bewegungsfertigkeit erzielt werden kann, die alternativen Behandlungstechniken überlegen ist.

Bewegungstransfer Wie verhält es sich dann mit dem Transfer einer (wieder-)erlernten Bewegung in eine andere? Wie im allgemeinen Kapitel zum motorischen Lernen von Bewegungsfertigkeiten schon angesprochen ist ein Transfer normalerweise sehr gering ausgeprägt und nur für sehr ähnliche motorische Aufgaben und Bewegungen zu erwarten. Kürzlich sind dazu aber Arbeiten veröffentlicht worden, die für die Neurorehabilitation Hinweise darauf liefern, dass es Transferleistungen aufgabenspezifischen Trainings der oberen sowie unteren Extremität auf andere Bewegungsaufgaben gibt:

- So stellten Hornby et al. [17] nach einem hochintensiven Gangtraining nach Schlaganfall fest, dass sich die Patienten unter anderem im Bereich des statischen Gleichgewichts verbessert hatten.

- Schaefer et al. [36] berichteten einen Transfereffekt von einer spezifischen trainierten Aufgabe auf zwei nicht trainierte Aufgaben. So waren Schlaganfallpatienten, nachdem sie in fünf Tagen insge- samt 2250-mal mit einem Löffel Bohnen von einer zu einer anderen Schüssel bewegt hatten (feeding task), auch besser bei den Aufgaben, kleine Würfel von einer Schachtel in eine andere Schachtel zu bewegen (vgl. Box and Block Test, sorting task) und Knöpfe zu schließen (dressing task). Die Autoren konnten zeigen, dass die Bewegung zwischen dem „feeding task“ und dem „sorting task“ sehr ähnlich war, während sich die beiden Aufgaben deutlich vom „dressing task“ unterschieden. Im Sinne der Ähnlichkeitshypothese beim Fertigkeitstransfer würde man einen höheren Transfer für die ähnliche Aufgabe und einen sehr geringen oder keinen Transfer für die nicht ähnliche Aufgabe erwarten. Die Ergebnisse zeigten aber eine Verbesserung in allen drei Aufgaben, wobei wie zu erwarten die Verbesserung in der trainierten Aufgabe (feeding) am größten ausfiel. Dagegen zeigten sich keine Unterschiede im Leistungszugewinn zwischen den beiden anderen Aufgaben.

- Ähnliche Resultate konnten auch nach einem Gleichgewichtstraining gezeigt werden [22]. In dieser Studie wurden drei verschiedene Interventionen miteinander verglichen, in denen unterschiedliche Gleichgewichtsaufgaben trainiert wurden. Nur in sehr spezifischen Testsituationen konnten nach der Trainingsphase gruppenspezifische Anpassungen gefunden werden, während es in allen anderen Tests zu ähnlichen Verbesserungen in den Trainingsgruppen kam. Dabei verbesserten sich die Teilnehmer nicht nur in verschiedenen Gleichgewichttests, sondern konnten z. B. auch ihre Gehgeschwindigkeit im Durchschnitt um etwa 13\% steigern. Diese Ergebnisse, nämlich die unabhängig von der Aufgabenähnlichkeit gefundenen Verbesserungen bei Schaefer et al. [36] sowie die zwischen den Trainingsgruppen vergleichbaren Verbesserungen bei Kramer et al. [22], deuten allerdings nicht zwangsläufig auf klassische Transfereffekte bei Fertigkeitsaufgaben hin. Vielmehr könnten Prozesse, die zu einer generellen Verbesserung der körperlichen Fitness beitragen, wie z.B. die Regeneration nach einem Schlaganfall oder die Verbesserung der konditionellen Voraussetzungen während eines stationären Reha-Aufenthalts, diese Befunde erklären.

\section{Auf der Basis der Grundlagenliteratur zu Fertigkeitstransfer sollte auch in der Neurorehabilitation von einer höchst aufgabenspezifischen Verbesserung bei Training der koordinativen Voraussetzungen ausgegangen werden.}

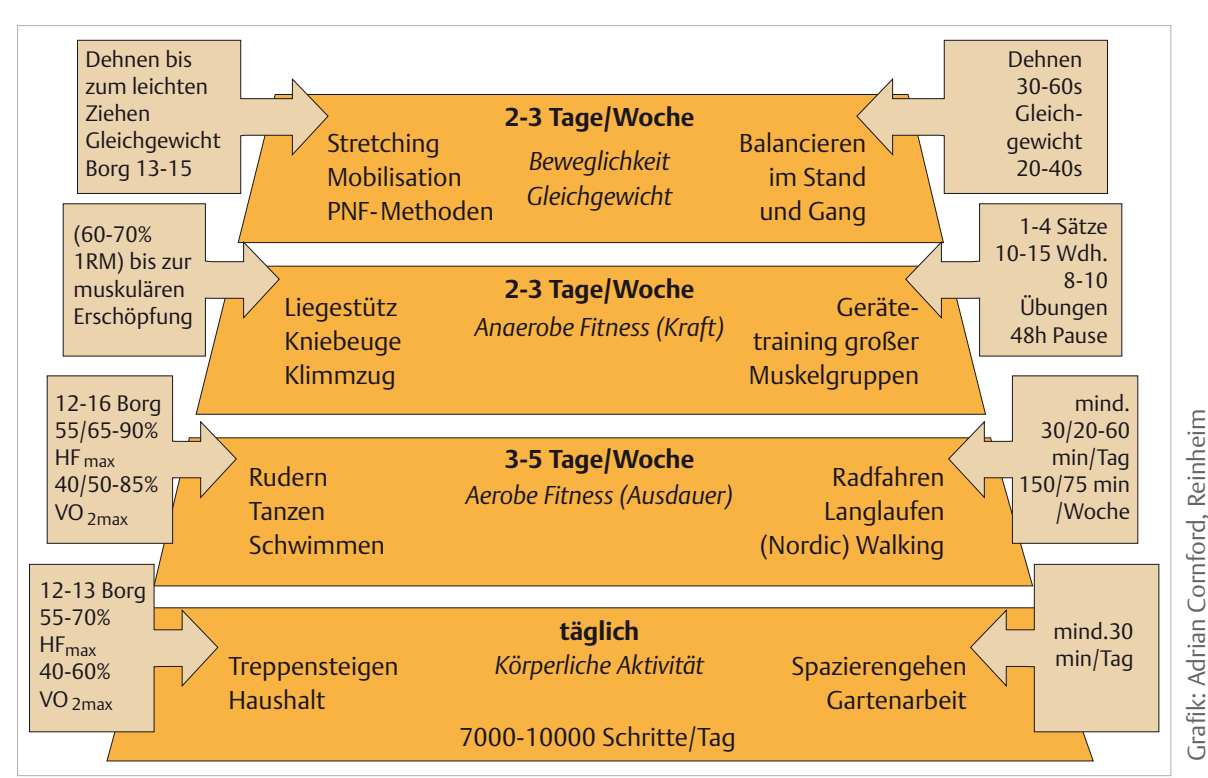

Abb. 4 Empfehlungen für ein gesundheitsorientiertes Training (Wochenplan). Aufbauend auf täglicher körperlicher Aktivität, sollte an drei bis fünf Tagen ein Ausdauertraining, an zwei bis drei Tagen ein Krafttraining und an weiteren zwei bis drei Tagen ein Dehn- und Gleichgewichtstraining durchgeführt werden [3, 42]. 


\section{Effektiv trainieren}

Im Bereich des Gesundheitssports existieren seit einigen Jahren evidenzbasierte Trainingsempfehlungen (s. Abb. 4). Nach diesen Richtlinien, die im Wesentlichen den Empfehlungen des American College of Sports Medicine und dem Swedish National Institute of Public Health entnommen sind [3, 42], würde der Trainingsumfang mit dem Ziel der Prävention von wohlstands- und altersbedingten Krankheiten, bei gesunden Personen pro Woche bei ungefähr 31/2 Stunden Alltagsaktivität, 21/2 Stunden Ausdauertraining bei geringer oder alternativ $75 \mathrm{~min}$ bei mittlerer bis hoher Intensität, 2-3 Stunden Krafttraining und 2-3 Stunden Dehnund Gleichgewichtstraining liegen.

\section{Insgesamt beläuft sich die empfohlene Trainingszeit zusätzlich zur Alltagsaktivität auf 7-10 Stunden pro Woche. Der größte Anteil entfällt dabei auf das Training der konditionellen und lediglich ein geringer auf das Training der koordinativen Voraussetzungen.}

Es ist offensichtlich, dass in der Neurorehabilitation nicht die konditionellen Leistungsvoraussetzungen, sondern in den meisten Fällen die Wiederherstellung von motorischer Funktion und damit die koordinativen Voraussetzungen im Vordergrund der Therapie stehen. Dementsprechend nehmen auch Physio- und Ergotherapie eine zentrale Rolle ein. Mit dem vorliegenden Überblicksbeitrag sollte explizit der ebenfalls hohe Stellenwert der motorischen Trainingstherapie für eine erfolgreich verlaufende Rehabilitation verdeutlicht werden. Nachfolgend werden die wichtigsten Kriterien eines effektiven Trainings noch einmal zusammengefasst dargestellt.

Effektiv trainieren bedeutet:

- konstitutionelle, konditionelle, koordinative und psychische Leistungsvoraussetzungen analysieren und deren Abhängigkeiten untereinander beachten
- das Training zielgerichtet, planmäßig und systematisch entwickeln, durchführen und analysieren

- zur Verbesserung der konditionellen Voraussetzungen die Belastungsnormative (Umfang, Intensität, Pausen) im Hinblick auf die Ziele und die individuellen Leistungsvoraussetzungen definieren

- zur Verbesserung der koordinativen Voraussetzungen fertigkeitsorientiert trainieren unter Beachtung der wissenschaftlichen Erkenntnisse, insbesondere zur Bedeutung von Bewegungswiederholung, KontextInterferenz-Effekt, Feedback und Instruktion sowie Aufmerksamkeit und Transfer

Die zu Beginn erwähnte Faustformel in der neurologischen Rehabilitation „repetitiv, intensiv, aufgabenspezifisch" kann demnach durch die oben angeführten Kriterien weiter substantiiert werden. Insbesondere sollte, trotz der Zielsetzung, die motorische Funktion wiederherzustellen, nicht vergessen werden, dass die konditionellen Voraussetzungen nicht nur weitreichende Auswirkungen auf die Gesundheit im Allgemeinen besitzen, sondern auch die spezifische Grundlage der motorischen Leistungsfähigkeit an sich darstellen. Da sich ein fertigkeitsorientiertes Training nachweislich höchst aufgabenspezifisch auswirkt, könnte eine verstärkte Einbeziehung von spezifischem Kraft- und Ausdauertraining zu einer Steigerung der allgemeinen „Fitness“ und zu einer Verbesserung der Rehabilitationsoutcomes in der Breite führen.

Die komplette Literaturliste finden Sie unter www.thieme-connect.de/ejournals

Markus Gruber ist Professor
für Sportwissenschaft und
Leiter des Sensorimotor
Performance Labs (SPL) an der
Universität Konstanz. Seine
Forschungsarbeiten beschäfti-
gen sich mit den biologischen
Grundlagen der körperlichen

\section{Bibliografie}

DOI 10.1055/s-0041-104793

neuroreha 2015; 7: 154-163

(c) Georg Thieme Verlag KG

Stuttgart · New York · ISSN 1611-6496 